\title{
A note on positive definite functions
}

by

NEIL W. RICKERT (Chicago)

It is known that for a locally compact group $G$ with left invariant Haar measure, if $\iint \varphi\left(x^{-1} y\right) f(x) \overline{f(y)} d x d y$ exists (i.e. the integrand is integrable) and is non-negative for every choice of $f$ in $L^{1}(G)$, then $\varphi$ is almost everywhere equal to a continuous positive definite function. In [2] the question is posed as to what happens if the hypothesis is modified to require the integral to exist and be non-negative for every $f$ in $L^{p} \cap L^{q}$ (where $1 / p+1 / q=1$ ). They construct an example to show that $\varphi$ need not be almost everywhere equal to a positive definite continuous function for non-discrete $G$. There construction depends on the idea that the integral positive definitess of $\varphi$ is reflected in the positivity of the Fourier transform of $\varphi$. They construct a $\varphi$ by looking at its Fourier transform but make sure that $\varphi$ is not an $L^{\infty}$-function. Their construction is essentially limited to groups which are either compact or Abelian since these are the groups for which Fourier analysis can most readily be used.

The purpose of this note is to suggest an alternative construction which avoids the use of Fourier analysis, and so applies to all non-discrete locally compact groups. Our argument, like that of [2], represents $\varphi$ as a limit of continuous positive definite functions.

Let $K$ be a compact set of positive measure. Choose the real number $M$ so that $1 / M$ and $M$ respectively are lower and upper bounds for the modular function of $G$ on the compact set $K$. Let $k$ be any real number such that $k \geqslant 1$. Let $U_{n}$ be a measureable subset of $K$ satisfying $0<m\left(U_{n}\right)=\varepsilon_{n} \leqslant 2^{-n}$, where $m$ denotes Haar measure on $G$. (It is here that we assume that $G$ is not discrete.)

Let $f_{n}$ be a measurable real-valued function on $G$ which vanishes outside $U_{n}$ and which satisfies $0 \leqslant f_{n}(x) \leqslant k / \varepsilon_{n}$ and $\int f_{n}(x) d x=1$. (For example we could take $f_{n}(x)=1 / \varepsilon_{n}$ if $x$ is in $U_{n}$. If $U_{n}$ is open and $k>1$, we could choose $f_{n}$ to be a continuous function, or even a $C^{\infty}$ whenever that makes sense.)

Writing $f_{n}=1 \cdot f_{n}$ and applying the Schwarz inequality yields $1 \leqslant\left\|f_{n}\right\|_{2}^{2} \varepsilon_{n}$ so $\left\|f_{n}\right\|_{2}^{2} \geqslant 1 / \varepsilon_{n}$. On the other hand, the function $f_{n}(x y)$ con- 
sidered as a function of $x$, has $L^{p}$-norm at most $7 i \varepsilon_{n}{ }^{(1-p) / p}$. Using the integral form of the Minkowski inequality it follows that if we define

$$
g_{n}(x)=\int f_{n}(x y) f_{n}(y) d y,
$$

then

$$
\left\|\varepsilon_{n} g_{n}\right\|_{p} \leqslant k M \varepsilon_{n}^{1 / p} .
$$

Thus, by comparison with a geometric series, we see that the series $\sum \varepsilon_{n} g_{n}$ converges in $L^{p}$ (for $1 \leqslant p<\infty$ ) and (since the terms are nonnegative) almost everywhere to a function $\varphi$. Evidently, $\varphi$ is in $L^{p}$ for $1 \leqslant p<\infty$. It is easily verified that each $g_{n}$ is a continuous positive definite function. It follows from the Lebesgue monotone convergence theorem that

$$
\iint \varphi\left(x^{-1} y\right) h(x) \overline{h(y)} d x d y
$$

exists and is a non-negative real number for every continuous $h$ with compact support.

It can easily be verified that $\varphi\left(x^{-1} y\right)=\varphi\left(y^{-1} x\right)$. Thus, using the integral form of Minkowski's inequality, if $h$ is in $L^{p}, \int \varphi\left(x^{-1} y\right) h(x) d x$ is a function in $I^{p}$ with norm at most $\|\varphi\|_{1}\|h\|_{p}$. If $p>1$ and $1 / p+1 / q=1$, then $\varphi$ is in $L^{q}$ and using Hölder's inequality $\int \varphi\left(x^{-1} y\right) h(x) d x$ is a function in $L^{\infty}$ with norm at most $\|\varphi\|_{q}\|h\|_{p}$. If, in particular, $1<p \leqslant 2$, so that $p \leqslant q<\infty$, then we can conclude from the convexity of the $L^{p}$-norms ([1], p. 524) that $\int \varphi\left(x^{-1} y\right) h(x) d x$ is in $L^{q}$ with norm at most $\left(\max \left\{\|\varphi\|_{1},\|\varphi\|_{q}\right\}\right)\|h\|_{p}$. Thus if $h_{1}$ is also in $L^{p}$ it follows (using Fubini's theorem) that $\iint \varphi\left(x^{-1} y\right) h(x) \overline{h_{1}(y)} d x d y$ exists and is bounded in absolute value by $\left(\max \left\{\|\varphi\|_{1},\|\varphi\|_{q}\right\}\right)\|h\|_{p}\left\|h_{1}\right\|_{p}$. Thus the hermitian bilinear form

$$
\left(h, h_{1}\right) \rightarrow \iint \varphi\left(x^{-1} y\right) h(x) \overline{h_{1}(y)} d x d y
$$

is continuous on $L^{p} \times L^{p}$.

In particular, $\iint \varphi\left(x^{-1} y\right) h(x) \overline{h(y)} d x d y$ is a continuous function of $h$ in $L^{p}$. Since it is non-negative for continuous $h$ with compact support, it is non-negative for all $h$ in $L^{p}(1<p \leqslant 2)$ by continuity.

Nevertheless $\varphi$ is not almost everywhere equal to a continuous positive definite function, because it is not in $L^{\infty}$. For the $L^{\infty}$-norm of $\varphi$ is at least as great as $\left\|\varepsilon_{1} g_{1}+\ldots+\varepsilon_{n} g_{n}\right\|_{\infty}=\varepsilon_{1} g_{1}(e)+\ldots+\varepsilon_{n} g_{n}(e)$ (the last equality since $g_{j}$ is continuous positive definite). But $g_{j}(e)=\left\|f_{j}\right\|_{2}^{2} \geqslant 1 / \varepsilon_{j}$ so $\|\varphi\|_{\infty} \geqslant n$ for arbitrary $n$.

Thus we have proved:

THEOREM. Let $G$ be a non-discrete locally compact group. There is a function $\varphi$ which is not in $L^{\circ}$ (and thus fails to be almost everywhere equal to a continuous positive definite function), but which is in every $L^{p}$ for $1 \leqslant p<\infty$ and for which the integral

$$
\iint \varphi\left(x^{-1} y\right) h(x) \overline{h(y)} d x d y
$$

exists and is non-negative for every $h$ which is in some $I^{p}(1<p \leqslant 2)$.

The theorem of [1] is a corollary:

COROLLART. There is a function $\varphi$ which is not in $L^{\text {o }}$ with the property that if $1 \leqslant p \leqslant \infty$ and $h$ is in $L^{p} \cap L^{q}$ (where $1 / p+1 / q=1$ ), then

$$
\iint \varphi\left(x^{-1} y\right) h(x) \overline{h(y)} d x d y
$$

exists and is non-negative.

Proof. Simply note that under these conditions $h$ is also in $L^{2}$ and apply the theorem.

Remark. If $G$ is metrizable (respectively a Lie group) and if the $U_{n}$ are chosen to be symmetric, a basis for the neighbourhoods of the identity, and such that $U_{n}^{2}$ is contained in a compact subset of $U_{n-1}$, then the series defining $\varphi$ is a finite sum in a neighbourhood of any point other than $e$, so that if the $f_{n}$ are chosen to be continuous (respectively $C^{\infty}$ ) functions, we see that $\varphi$ is continuous (respectively $C^{\infty}$ ) except at $e$.

\section{References}

[1] N. Dunford and J. T. Schwartz, Linear operators $I$, New York 1958.

[2] E. Hewitt and K. A. Ross, Integrally positive definite functions on groups, Studia Math. 31 (1968), p. 145-151.

UNIVERSITY OF ILLINOIS AT CHICAGO CIRCLE

Reçu par la Rédaction le 8. 10. 1969 